\title{
bag-of-marbles: a Drosophila gene required to initiate both male and female gametogenesis
}

\author{
Dennis M. McKearin ${ }^{1}$ and Allan C. Spradling \\ Howard Hughes Medical Institution Research Laboratories, Carnegie Institution of Washington, Baltimore, Maryland 21210 \\ USA
}

\begin{abstract}
In Drosophila, male and female gametes begin development when a stem cell divides to produce a cyst precursor. Subsequently, four special divisions give rise to a cluster of 16 interconnected cystocytes that develop into a single egg or 64 sperm. We identified and characterized a gene, bag-of-marbles (bam), that disrupts cyst formation in both sexes. An apparent null mutation causes abnormal cysts to form containing an excess number of cells that cannot differentiate into gametes. bam function resides within a simple 2.2-kb transcription unit encoding a single 442-amino-acid protein that shows similarity to the product of the ovarian tumor gene. The specific expression of bam RNA within female cystoblasts suggested that it might be involved in the specific cell-cycle alterations that occur during cystocyte divisions.
\end{abstract}

[Key Words: Drosophila development; cyst formation; gametogenesis; bag-of-marbles]

Received August 31, 1990; revised version accepted October 18,1990.

Stereotyped patterns of cell division often accompany differentiation (see Horvitz 1988). Recently, considerable progress has been made in understanding how the basic events of the eukaryotic cell cycle are regulated and particularly how cells are driven into mitosis /for review, see Murray and Kirschner 1989). However, relatively little has been learned about the programming of specialized cell cycles, nor is their developmental significance well understood. The production of 16-cell cysts during early gametogenesis in Drosophila melanogaster provides a favorable system for identifying genes that control specialized cell cycles.

The early stages of Drosophila gametogenesis are remarkably similar in the two sexes /for review, see Lindsley and Tokuyasu 1980; Mahowald and Kambysellis 1980). In both ovaries and testes, germ-line stem cells are sequestered at the anterior end of the organ. When a germ-line stem cell divides, one of the daughters remains attached and continues to function as a stem cell, whereas the other daughter becomes the "founder" of a 16-cell syncytial cyst. Four successive divisions of this cystoblast or spermatoblast /within the ovary or testis, respectively/ produce a clone of 16 sister germ line cells that remain interconnected due to an unusual process of incomplete cytokinesis that generates interconnecting ring canals. In the ovary, a complement of somatic cells encloses each 16-cell cyst sometime after it is formed as it passes through a specific region of the

'Present address: Department of Biochemistry, University of TexasSouthwestern Medical Center, Dallas, Texas 75235 USA. germarium. In contrast, during spermatogenesis, the founding spermatoblast is enclosed by two somatic cells prior to the four spermatoblast divisions. Nonetheless, both processes generate cysts of 16 germ-line-derived cells surrounded by a layer of somatic cells. Shortly thereafter, the pathways diverge; female cysts go on to produce a single egg, whereas male cysts produce 64 spermatids.

These morphological parallels suggest that oogenesis and spermatogenesis may share genetic functions utilized in cyst production. Such genes would be needed to produce germ line founder cells, to program the four blast cell divisions, to limit cytokinesis and generate ring canals, and to mediate enclosure of cysts within an appropriate somatic cell layer. Genes controlling cyst production might be identified in two ways. Mutations should simultaneously produce both male and female sterility. Furthermore, abnormalities in gametogenesis should begin very early, corresponding to the time of cyst production. Extensive genetic searches for mutations disrupting male or female fertility have been carried out in Drosophila (see Lindsley and Zimm 1985, 1990|. Although some candidate mutations that might define early cyst functions have been generated, including benign gonial cell neoplasm (bgcn; Gateff 1982), none have been characterized in detail. In contrast, a class of "tumorous egg chamber" genes required solely in the female for cyst formation, particularly the ovarian tumor gene $(o t u)$, have been analyzed more thoroughly (King and Storto 1988; Steinhauer et al. 1989). These mutations produce ovarioles with irregular proliferating 
cells and abnormal cysts. Recently, studies of several loci that produce "tumorous" ovarian defects, including Sex-lethal (Sxl) (Schüpbach 1985; Steinmann-Zwicky et al. 1989), sans fille (snf) (Oliver et al. 1988; SteinmannZwicky 1988), and ovo (Oliver et al. 1990) have suggested that some of these genes may interfere with the process of germ line sex determination \{see Nöthiger et al. 1989).

We used single P-element insertional mutagenesis (Cooley et al. 1988) to generate a collection of mutations disrupting Drosophila gametogenesis. In this report we describe a new gene, bag-of-marbles (bam), that encodes a function common to both male and female cyst production. Our experiments argue that bam may regulate cystoblast cell divisions and suggest a new hypothesis concerning the origin of tumorous egg chambers.

\section{Results}

Isolation of a new mutation, bam

Among a collection of 1317 lines bearing single P-element insertions (Cooley et al. 1988), we identified a recessive mutation that sterilized both males and females. This stock carried a single $\mathrm{P}$ transposon in polytene band $96 \mathrm{C}$ on the right arm of chromosome 3 (data not shown). Df(3R)L16, a deletion of polytene bands 96A-96E /Gonzalez et al. 1989), failed to complement the sterility in both sexes, suggesting that the mutant phenotype was caused by the insertion. Consequently, we examined the phenotype in gonads from adult homozygotes to determine whether this mutation might disrupt an early process of gamete development in both sexes.

Homozygous bam females were fully viable but did not lay eggs. Microscopic examination of the ovaries from such females revealed that oogenesis was disrupted at very early stages of development. Each of the tubular ovarioles within a wild-type ovary contains, at its anterior end, a germarium, where egg chambers are first assembled, and seven to eight egg chambers in successively older stages of oogenesis. bam ovaries remained small, because they contained ovarioles with abnormal germaria and only one to three small abnormal egg chambers. In a wild-type germarium, 16-cell cysts are produced and enveloped with follicle cells (Fig. 1A, bracket). bam germaria, like those of previously described tumorous mutants, contained a disorganized mass of cells and assembled cysts containing 50 to several hundred cells (Fig. 1B, bracket). Cysts assembled in bam ovaries usually contain 50-100 cells and often contain many hundreds. Very rarely, a few cells within a bam chamber attained a large size reminiscent of pseudonurse cells, a second phenotype observed in several tumorous ovary mutants (see King 1970). There was little difference between the phenotype of bam homozygotes and $\mathrm{bam} / \mathrm{Df}(3 \mathrm{R}) \mathrm{L} 16$ females (Table 1), suggesting that the insertion mutation was a strong hypomorph or null allele.

Examination of bam testes suggested that gametogenesis was also disrupted at an early stage in males. These testes contained abnormal cysts populated with
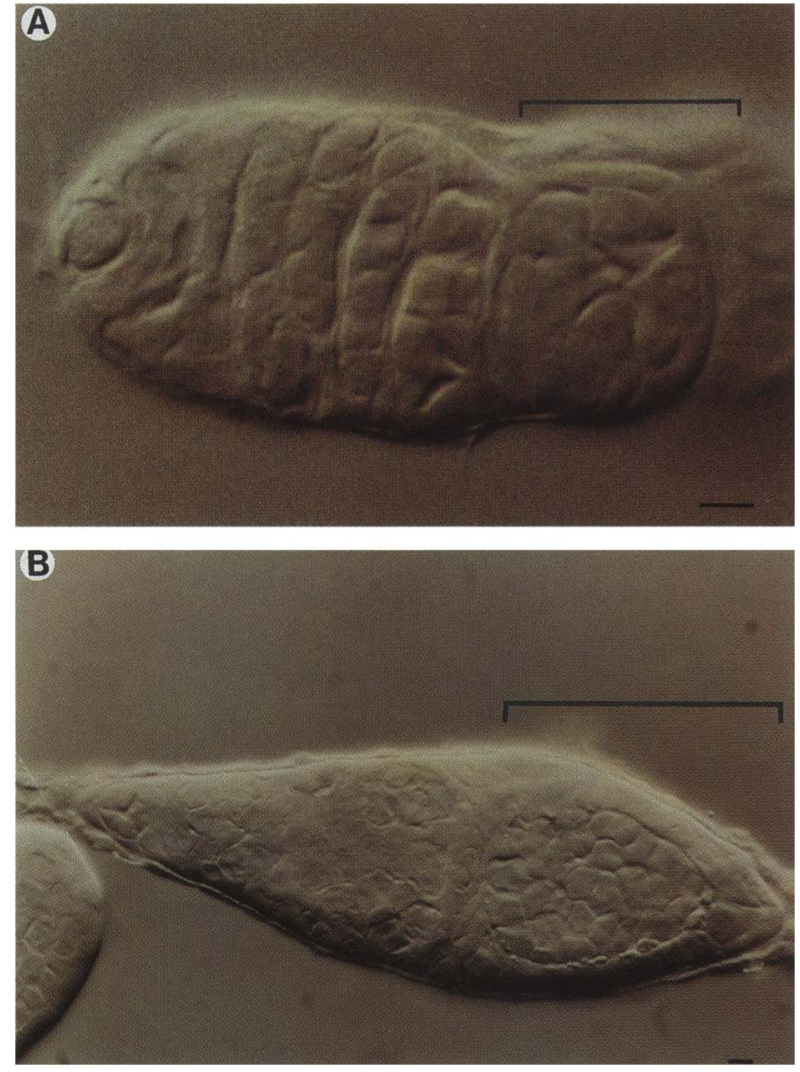

Figure 1. bam ovaries contain tumorous egg chambers. In each plate, anterior is to left. $(A)$ Micrograph of a wild-type germarium. The bracket indicates a fully formed egg chamber with 16 cystocytes enclosed by a layer of follicle cells. $|B|$ Representative example of a bam ovariole showing the tumorous ger marium and egg chamber. Bar, $10 \mu \mathrm{m}$. Note that the magnification scales of bam and wild type are different by a factor of 2.4

an excessive number of small cells the size of primary spermatocytes. Normally, the 16 primary spermatocytes within a cyst increase 25 -fold in size prior to the onset of meiosis (cf. Fig. 2A,B). However, bam spermatocytes remained about the size of early spermatocytes (Fig. 2C). They never underwent subsequent morphological changes characteristic of meiosis and spermiogenesis. Consistent with an early developmental arrest, expression of two genes normally induced during primary spermatocyte growth, $\beta 2$-tubulin (Kemphues et al. 1982; $\mathrm{Mi}$ chiels et al. 1989) and mst325 (DiBenedetto et al. 1988), was not detected on Northern blots of RNA from mutant testes (data not shown). A final unusual feature of the bam spermatocyte cysts was their progression into a highly refractile state (Fig. 2D). Much of the lumen of the testis became filled with these refractile cysts in the mutant.

\section{Cloning the bam locus}

The production of cysts with an increased number of cells in both male and female gonads was consistent with a role for the bam gene in controlling cystoblast 
Table 1. Deficiency testing bam phenotype

\begin{tabular}{lcccc}
\hline & $\begin{array}{c}\text { Number of } \\
\text { females }\end{array}$ & TC & PNC & $15+1$ \\
\cline { 3 - 5 } bam $/ \mathrm{bam}$ & 16 & 420 & 0 & 0 \\
$\mathrm{bam} / \mathrm{Df}$ & 17 & 446 & 1 & 0
\end{tabular}

These data test $D f(3 R) L 16$ for complementation of bam and test whether the insertion bam allele behaves genetically as a null mutation by comparing the effect of one copy versus two copies of the mutant bam allele. Animals were kept at $23-25^{\circ} \mathrm{C}$, and their ovaries were dissected 3-6 days after eclosion. The ovarioles of dissected ovaries were teased apart, and the chambers within were scored at $40 \times$ in Normarski optics on an Optiphot microscope. The number of discrete chambers that contained 15 nurse cells +1 oocyte $(15+1)$, pseudonurse cells (PNC), or only tumorous cells (TC) was tallied as a measure of the severity of the effects of the two genotypes. The number of females that produced the ovaries from which these egg chambers were recovered is given.

proliferation. We therefore undertook molecular studies. P-element sequences were used as a molecular tag to recover a genomic fragment flanking the insertion site. A $60-\mathrm{kb}$ region was subsequently recovered from a cosmid library of wild-type genomic DNA. Figure 3 presents the structure of a $5.5-\mathrm{kb}$ BamHI fragment from this region that surrounds the site of transposon insertion.

To identify RNAs that might correspond to bam tran- scripts, a series of genomic DNA fragments derived from the $5.5 \cdot \mathrm{kb}$ fragment were used to probe Northern blots of poly $(\mathrm{A})^{+}$RNA from wild-type male and female flies (data not shown). Four transcripts derived from the region adjacent to the site of insertion were mapped at low resolution (Fig. 3). Three overlapping RNAs, $\gamma, \delta$, and $\epsilon$, were transcribed toward the left of the insertion site, whereas the fourth, $\alpha$, was transcribed from the opposite strand.

To associate the bam gene with one or more of these transcripts, transformation rescue experiments with two constructs were carried out. P/C-ry-a] encompassed the entire rightward transcript, but truncated $\gamma, \delta$, and $\epsilon$. The $P / C-r y-; \delta \varepsilon]$ transposon truncated the rightward RNA but included all fragments that had labeled $\gamma, \delta$, and $\epsilon$. A germ-line transformant of each construct was tested for the ability to complement bam male and female sterility. $P[C-r y \cdot a]$ rescued gamete morphology and sterility in both sexes, whereas $P / C-r y-\gamma^{\prime} \delta \varepsilon /$ had no effects on fertility or gonadal morphology. We concluded that the 2.9-kb genomic region within $P / C$-ry-a] contained the fully functional bam gene and that the $\alpha$ transcript was a strong candidate to encode bam function.

To examine the expression of the putative bam transcript in more detail, RNA was prepared from gonads and gonadectomized carcasses of wild-type and mutant adults and analyzed on Northern blots (Fig. 4A). Under the conditions of hybridization used, the $2.0-\mathrm{kb}$ putative
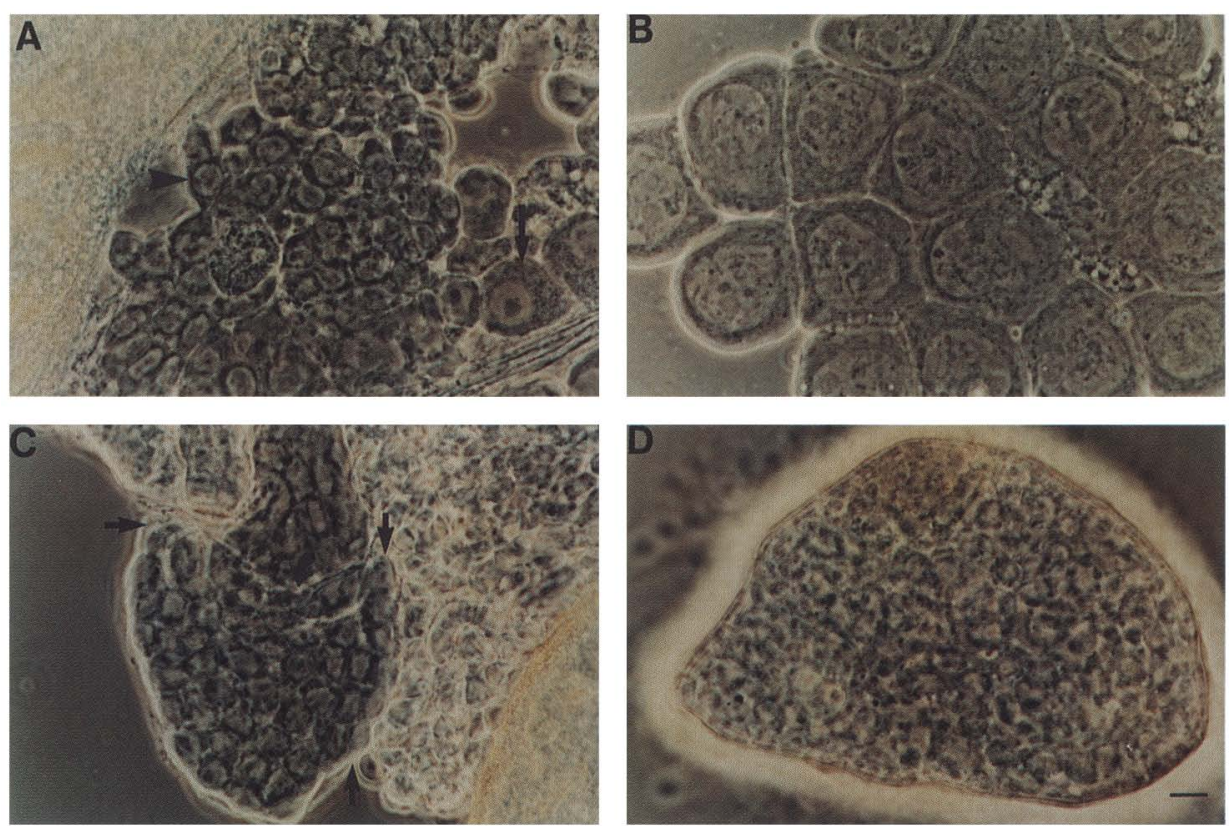

Figure 2. bam spermatocytes arrest during early spermatogenesis. $\mid A$ and $B \mid$ Spermatocytes from wild-type testes; $\mid C$ and $D \mid$ spermatocytes from bam testes. (A) Examples of early (arrowhead) and mid-stage (arrow) primary spermatocytes that have been released by manual disruption from wild-type testes. $(B)$ A 16 -cell cluster of late stage primary spermatocytes. Note the difference in size between early $(A)$ and late stage $|B|$ spermatocytes reflecting the accumulation of transcribed and translated gene products during the growth phase of spermatocyte development. $(C$ and $D)$ Examples of spermatocyte cysts found in bam testes. The boundary of an intact cyst is indicated by arrows in $C$ for clarity. Note that the cells contained within this cyst are approximately equal in size to early primary spermatocytes. The highly refractile cells that characterize end-stage bam cysts are shown at right of the outlined cyst. A dramatic example of such a cyst is presented in $D$. In both $C$ and $D$, bam cysts contain $>16$ spermatocytes. All micrographs are taken in phase contrast at the same magnification $(330 \times)$. Bar in $D, 10 \mu \mathrm{m}$. 


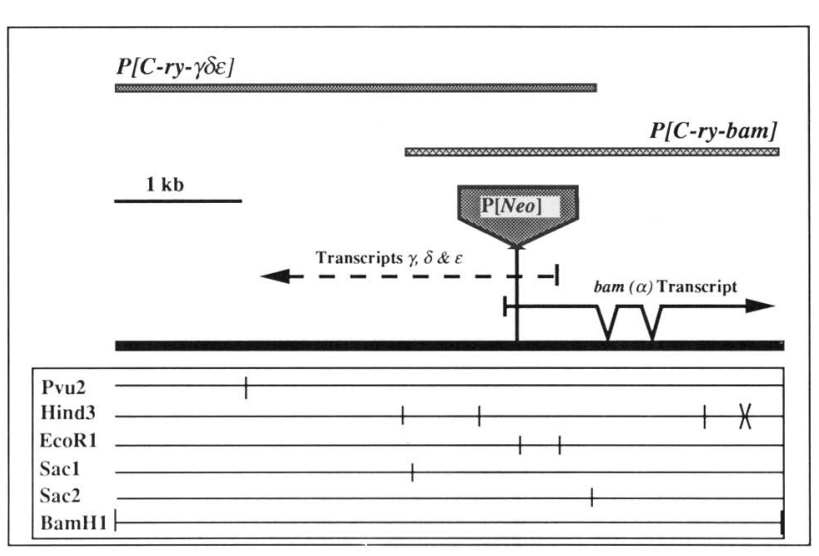

Figure 3. Chromosomal region of the bam gene. A restriction map for the wild-type bam locus is shown together with the position of the 5.2-kb P/pUC-hs-Neol transposon in the bam mutant. The relative positions and orientations of the bam transcript and the family of overlapping mRNAs, $\gamma, \delta$, and $\epsilon$, are also shown; positions of introns are known for bam and are shown. The $\gamma-\epsilon$ transcripts, which do not rescue bam, have not been mapped as accurately and are therefore represented as a broken line, which indicates the genomic DNA that they span; hybridization experiments reveal that much of this DNA encodes introns. (Top) A representation of the DNA fragments used to construct the transposons that test for transformation rescue of bam phenotype.

bam RNA, as well as a smaller $0.7-\mathrm{kb}$ species, was observed in wild type. The smaller RNA is due to limited homology between the probe and an RNA encoded elsewhere because it was never observed when more stringent hybridization conditions were employed. The 2.0$\mathrm{kb}$ transcript was present in both wild-type ovaries and testes but could not be detected on even very long expo- sures of RNA from adult carcasses. The 2.0-kb RNA was expressed throughout oogenesis and in 0- to 2-hr embryos but was absent by $4 \mathrm{hr}$ of embryonic development (Fig. 4B). Because the 2.0-kb transcript was specifically expressed in the tissues affected by the mutation, and because no other transcripts encoded within the $2.9-\mathrm{kb}$ rescuing fragment were detected, we concluded that the $2.0-\mathrm{kb}$ transcript encodes bam function. In the bam mutants, both ovaries and testes contained an RNA that appeared to be slightly larger than the normal $2.0-\mathrm{kb}$ transcript; the molecular basis for the altered mobility of the mutant RNA has not been determined.

\section{Structure and sequence of the bam gene}

Once the authentic bam gene had been identified, we analyzed its structure in detail (Fig. 5). A 1571-bp cDNA was recovered from an ovary cDNA library and its sequence determined, including an 8-bp poly(A) tail not encoded in the genome. In addition, genomic sequence beginning at a HindIII site $\sim 400$ bp upstream from the site of insertion and continuing into the region defined by the cDNA was determined. The start site for the bam gene and the location of introns throughout the gene were identified by RNase protection experiments that covered the entire region encoding the 2.0-kb RNA (not shown). The bam transcript spanned $\sim 2160 \mathrm{bp}$; it initiated $51 \mathrm{bp}$ upstream from the site of transposon insertion and contained two small introns, yielding an mRNA of 1990 nucleotides, close to the 2.0 -kb size observed on Northern blots. The first AUG codon is followed by only five amino acid codons prior to a terminator. However, the second AUG codon at position +180 , which, unlike the first site, corresponds closely to the nucleotide consensus sequence for Drosophila initiation

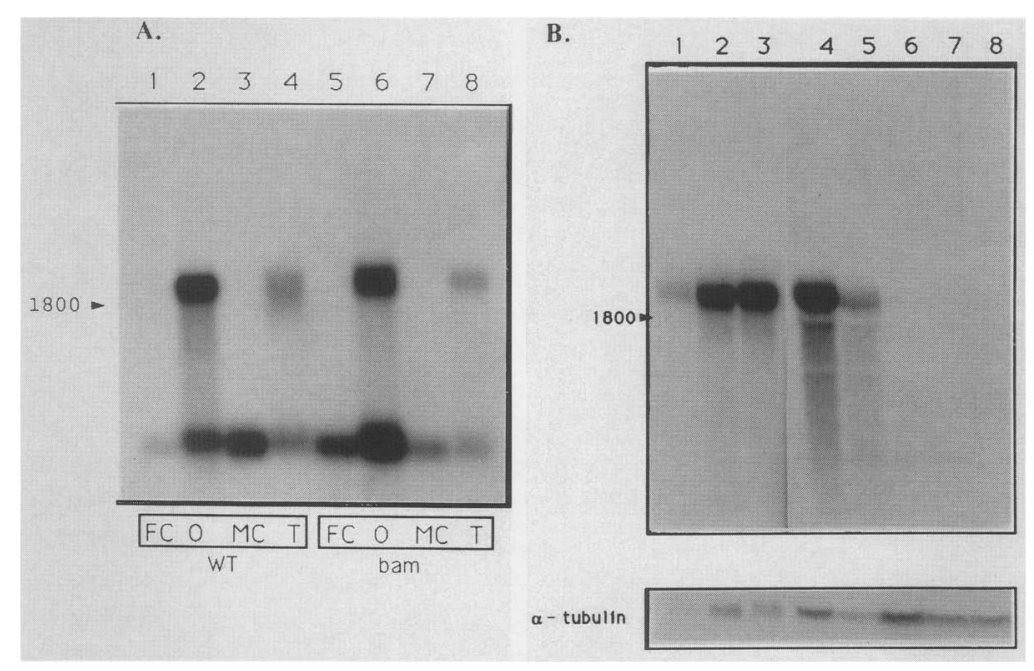

Figure 4. Distribution of bam transcript. (A) Hybridization of antisense bam probe to poly $(\mathrm{A})^{+}$RNA from the dissected tissues of wild-type (WT) or bam animals. The mRNA detected at $\sim 2000$ nucleotides corresponds to bam mRNA; the lower molecular weight transcript is a cross-hybridizing species whose identity is unknown. Higher stringency hybridization conditions eliminate this cross-hybridization (e.g., see Fig. 4B). (F) Female animal RNA; (M) male animal RNA; $(\mathrm{FC})$ ovarectomized female carcass; $(\mathrm{O})$ ovary; (MC) gonadectomized male carcass; $(T)$ testis. $(B)$ Egg chambers isolated from wild-type females that had been fed an enriched diet for 24-48 hr prior to dissection were staged according to the stages of King (1970). Egg chambers of germarium stage through stage 8 were collected from females $<16 \mathrm{hr}$ old because these animals had not yet begun to produce vitellogenic stages. Embryo RNA was isolated as described in Materials and methods. Following hybridization with bam probe, the probe was removed and the membranes reprobed with a radioactively labeled DNA for the ubiquitously expressed $\alpha 1$-tubulin (kind gift of $L$. Kalfayan) to verify mRNA integrity in each lane (shown at bottom). The order of the RNA loaded in a lane is germaria, stage 8 (lane 1); egg chamber, stages 9-11 (lane 2); egg chamber, stages 12-14 (lane 3); embryonic development, 0-2 hr (lane 4); embryonic development, $2-4 \mathrm{hr}$ (lane 5); embryonic development, 4-8 hr (lane 6); embryonic development, 8-16 hr (lane 7); embryonic development, 16-24 hr (lane 8). 
Figure 5. Sequence of the bam- gene. The bam gene sequence, together with 379 bp of sequence at the $5^{\prime}$ end of the gene, is shown. Sequences of the two introns are included in lowercase letters. The numbers at left refer to nucleotides; those at right refer to amino acids of the predicted protein. Nucleotide numbers are presented only for bases that appear in the mature bam transcript and therefore do not include the intron nucleotides. The putative start site of transcription, based on RNase protection assays, is indicated as +1 . An arrow at +51 indicates the nucleotide that lies to the $5^{\prime}$ side of the site of insertion of the mutagenic transposon in the bam mutant. A putative TATA-box sequence at -31 is indicated in underlined italics. The ATG at position +186 initiates the open reading frame (ORF) for the putative bam protein, the predicted sequence of which appears as a oneletter code over the central nucleotide of the corresponding codon; this ORF terminates with a codon ending at +1514 . A poly $(\mathrm{A})$ addition sequence (boldface) lies 23 nucleotides upstream of the end of the transcript.
-379 AGCTTATCGTCGTCAGAATTACAAAAACAAAT TGAATATGAAAATGGGTTATTGCTACTTCATTATTATTGTCAC -304 GATATATGATAATTTATACAAAATGTGATAAATCCCAAATTGTTAAATAATGCTTTGGCTTGCTTTATACAAAC -229 CACTAGATAATTAAAATATAGGTGGCCTAAATTGTTGCATGTTGTTTTATAATTAATCAGCAATTTGATTTGGTT -154 GTGATCGACCAAATCAGTGTGTATAATTGTAGTTAAAATGTAAAGCTTCGTAATGGATTATTGAATCGCATTTCA

-154 GTGATCGACCAAATCAGTGTGTATAATTGTAGTTAAAATGTAAAGCTTCGTAATGGATTATTGAATCGCATTTCA
-79 AATTTCTTAAAATGCGCCCGGGTCARTGACCTTTTGAGGTGACCATAAATTGAAACTTATTTGTGCGACGGCAAC $+1$

CCTGTTCTGGGTACTCGACATGATATCGATACGTTAACAACAAAGAGTCTGGACGCCATCATTCTTCCTCTTTCT CCTGAATTCGCAGACAGCGTGGCGTCAGCGATTTCAAACGGT AAAAAGAACCTGGCGATAAGGAAAGATTT TAAAA 146 GGCAAAAATCGAGTGATTTGTGTGATTTAACTTAAGAATAATGCTTAATGCACGTGACGTGTGTCCTGAGGGCAA $\begin{array}{lllllllllllllllllllllllll}D & D & Q & Q & L & D & H & N & F & K & Q & M & E & E & H & L & A & L & M & V & E & G & N & E & N\end{array}$ CGACGACCAGCAGT TGGACCACAA. TTAAGCAGATGGAGGAGCATT CGAAGATCCGAGGAAAGCCACTTGTGAGTACGAGGATACGAACGAAGATGGTGCAACCTGCACATCGGGGTTTT $\begin{array}{lllllllllllllllllllllllll}S & E & I & Q & E & N & F & G & R & L & R & L & C & D & V & T & A & P & L & L & E & F & H & G & L\end{array}$ ATCCGAAATCCAGGAGAACTTCGGTAGACTCCGGTTGTGTGACGTTACTGCACCACTCCTCGAATTCCACGGTT T $\begin{array}{llllllllllllllllllllllllll}D & C & L & Q & Q & I & Q & K & R & S & R & I & L & H & S & T & V & L & R & P & R & S & R & D & P\end{array}$ GGATTGCTTGCAACAGATTCAAAAGCGCTCGCGCATTTTCCATTCGACGGTTCTCCGGCCAAGAAGTCGCGATCC

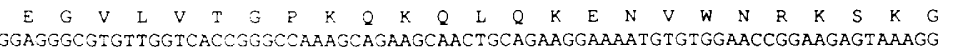

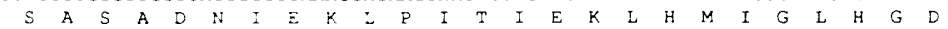
CTCTGCGTCCGCGGATAATAT :GAGAAACTGCCCATAACTATTGAGAAACTGCATATGATTGGTCTGCACGGCGA

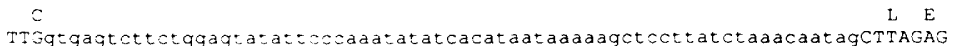

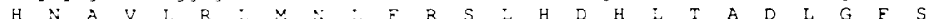
CAchacoCCOnOCnGComman CACAACOCCOTGCTCS CGCCAAAAC $C$ CAATGCCC.CGAACARC. $R$ $Y$ Q L Q V V R

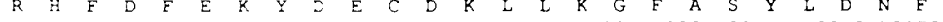
CGCCACTTCGATTICGAGAAATACUACSAGTGTGACAAGTTGCTTAAGGGTITCGCATCCTATTTGGACAACTTC $\begin{array}{lllllllllllllllllllll}K & L & L & L & K & P & K & M & F & N & R & N & G & N & S & G & S & N & A & D & K\end{array}$ AAACTGCTITTAAAGCCCAAAATGCGCAATCGAAACGGAAACTCGGGGAGCAATGCGGACAAGTgt a agctgt ag

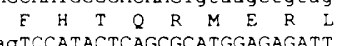

$204 \hat{4}$ atttgcaagcaaccattcageta: $5 c t g c a a c g a t t t t a t t a t t t a c a g T C C A T A C T C A G C G C A T G G A G A G A T T$

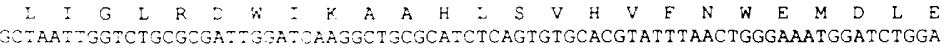

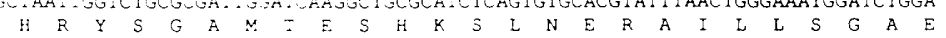
GCACCGCTACTCCGGGGCCATGACCSAAAGCCACAAGTCGTTGAACGAGCGGGCCATCCTTTTGTCCGGTGCCGA G R A A E A R G I S A E D L F I A Q R Y K L G G CTAAGGGCGGCCGAAGCGCGTSGAATCAGTGCGGAGGATCTGTTCATCGCCCAGAGATACAAACTGGGAGGTCC

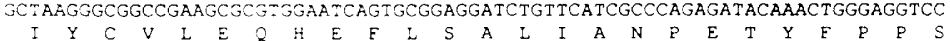
GATCTATTGCGTTCTGGAGCAGCATEAGTTCCTCTCCGCTCTGATCGCCAATCCAGAGACCTATTTCCCGCCCAG

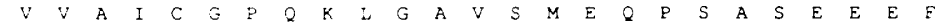
-GTTGTCGCCATT TGCGGGCCACAGA.AGCTTGGCGCAGTGAGCATGGAGCAGCCGTCAGCGTCGGAGGaGGaGT

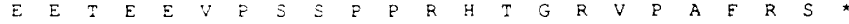

GAGGAGACCGAGGAag:GC DATCATCGCCACCTCGTCACACCGGACGTGTACCTGCCTTCAGAAGCTAAACTAA TOCTGTGCACATCGA AAAMOART GA GG AAAATATGCAATTTAAAAAGGCTACTCTTCTCATGGGAAGCAATAA

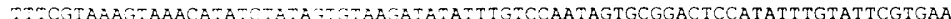
Ta com

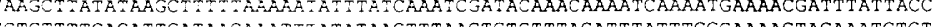
CCTGTTITGA TTGCTTTACACT TTATARACCTGTAOTTTACTTAAACTT FTGCAACTACGTTTTAT\%GTATGEGGAACTTACCAGTTTTTCTTATTTGCTTTGCATTTGTATTTTGAAAGTC
AAATAAATATTTACGATTGTGTT:GGACT.pOLY-A

\section{2}

codons (Cavener 1987), is followed by a 442 -amino-acid open reading frame that predicts a conceptual protein of $49 \mathrm{kD}$. This polypeptide contains an amino acid sequence at positions 404-432 that meets the criteria for a PEST sequence (Rogers et al. 1986). PEST sequences have been correlated with protein instability; the predicted bam protein carries a potential proteolytic cleavage site at residues $234-235$. To complete the transcription unit, the mRNA contains a 3 '-untranslated region of 480 nucleotides that ends 23 nucleotides downstream from a consensus poly $(\mathrm{A})$ addition signal.

When the predicted polypeptide sequence for bam protein was used to search the Swiss Protein Database, the highest scoring match was to the protein from another Drosophila locus that mutates to produce tumorous egg chambers, the otu gene (Steinhauer et al. 1989). Weak similarity was detected within three regions spanning $\sim 150$ amino acids in the central region of the bam protein (Fig. 6). About $20 \%$ of the residues were identical and $15 \%$ corresponded to conservative replacements within these regions. This degree of similarity does not necessarily indicate shared function, and the biochemical roles played by both the bam and otu gene proteins remain unknown. However, this finding suggests that there may be some relationship between the tumorous egg chamber defects produced by bam and otu mutations.

\section{bam is expressed specifically in early ovarian cysts}

If bam functions during cystocyte divisions, we might expect it to be specifically expressed during this stage in the germarium. To test this prediction, we characterized bam transcript expression by hybridization of digoxigenin-labeled antisense bam RNA probes to whole wildtype ovaries and embryos (Tautz and Pfeifle 1989). Figure 7, A and B, shows that bam mRNA is present at high levels in only one or two cells located near the anterior tip of the germarium. Following a stack of $\sim 10$ somatic "terminal filament" cells, germaria contain two or three large germ-line stem cells, and then more posteriorly, one or two cystoblasts and two-cell cysts. The hybridization we observed was always located in germ-line cells immediately posterior to the stem cells. Thus, bam is expressed in cystoblasts and/or very early cystocytes. A much lower level of RNA was detected in older cysts within the germarium.

Transcription from the bam gene appeared to be repressed in maturing egg chambers because no labeling could be detected in subsequent stages of oogenesis (Fig. 

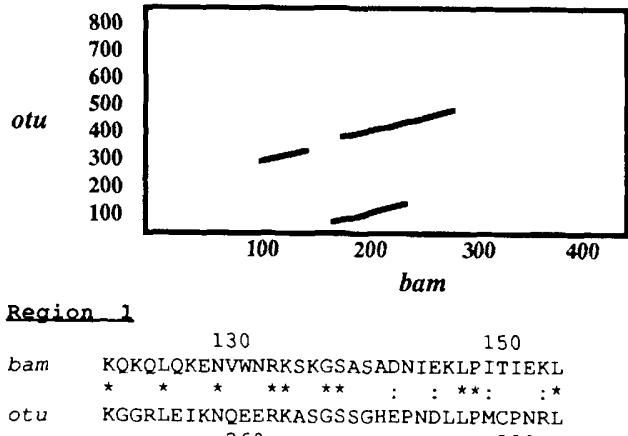

otu KGGRLEIKNOEERKASGSSGHEPNDLLPMCPNRL 260 280
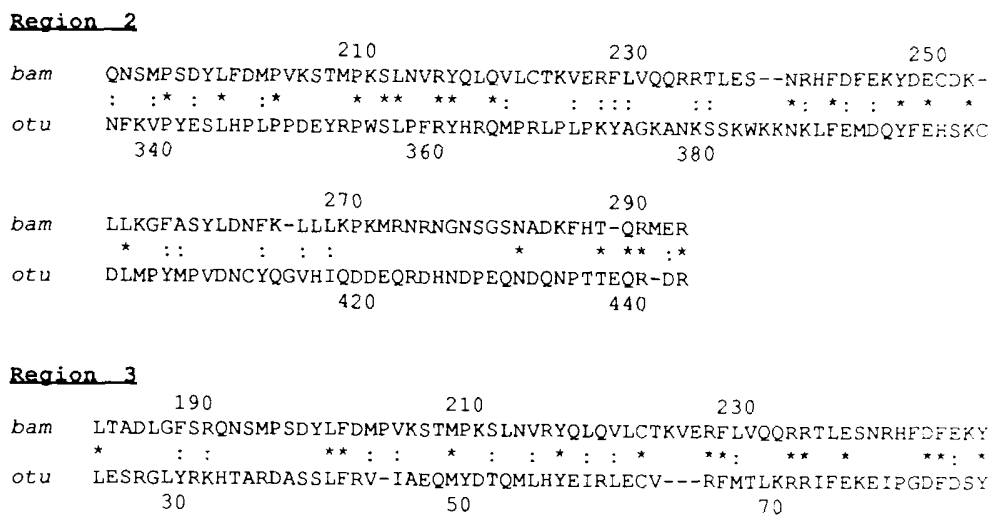

Figure 6. Comparison of the proteins predicted by the clones for the otu and the bam gene. When bampredicted protein sequence was used for comparison to other protein sequences deposited in the Swiss Protein Database, the highest scoring match was achieved with comparison to the otu protein. The protein sequence for the otu protein was recovered from the Swiss Protein Database and was compared to our sequence for the bam protein in the Staden program on the JHU VAX in matrix analysis format. The $x$-axis shows numbers of amino acids of the predicted bam protein; the $y$-axis is the same for the otu protein (Steinhauer et al.). Alignments were carried out on the protein sequence alignment program on the JHU VAX computer. The numbers in parentheses indicate the number of identical residues and conservative replacements, respectively, in the stretch of amino acids indicated. Asterisks (*) indicate identities, whereas colons $(:)$ indicate conservative replacements. Conservative replacements were scored as follows: |L-M-A-I-V; K-R; D-E; S-T; Y-W-F; $\mathrm{N}-\mathrm{Q}$. $\mathrm{H}, \mathrm{G}, \mathrm{C}$, and $\mathrm{P}$ were not scored as conservative replacements for any residues.
7C) until midway through stage 10, when high levels of RNA were observed in nurse cells (Fig. 7D). This RNA was transferred to the oocyte at the time of nurse cell breakdown and persisted in the mature egg and the early embryo (Fig. 7E). However, by gastrulation ( $3.5 \mathrm{hr}$ ), bam RNA could no longer be detected. The pattern of expression observed in situ thus supported the results of Northern blots (Fig. 4B).

\section{Discussion}

bam is required very early to form both male and female cysts

The bam mutation disrupted both male and female gametogenesis at very early steps. The earliest sign of egg development, the formation of interconnected cysts of germ-line cells, was blocked. Primary spermatocyte differentiation in the testis was also aberrant and appeared to stall prior to meiosis. In both cases, abnormal cysts formed, containing an excess number of cells that did not continue to differentiate. The similarity between the defects in both male and female gonads suggested that bam plays a role common to gametogenesis in both sexes. Consistent with this interpretation, the $2.0-\mathrm{kb}$ bam mRNA encoding a single 442 -amino-acid protein was found to be expressed in both gonads.

The cystoblast divisions in both ovaries and testes differ from the stem cell divisions that precede them. Cystoblasts and spermatoblasts divide exactly four times, whereas stem cells can divide indefinitely. The cystoblast contractile rings produce ring canals that maintain direct cytoplasmic contact between daughter cells. Finally, at least in the female, cystoblast divisions are cleavage-like because individual cells in 16-cell cysts are smaller than the precursor cystoblast. bam may be required to bring about these cell-cycle alterations. Because bam RNA appeared shortly after the stem cell division that produces each new cystoblast, the character, and possibly the number, of subsequent cystocyte divisions may be programmed at this time by the bam product in the cystoblast. The transient expression of bam mRNA and the fact that the protein carries a sequence that implies rapid turnover are consistent with the hypothesis that bam functions as a titratable counting factor during cystocyte divisions. In the absence of bam protein, stem cell daughters would be unable to modify their cell cycles following division, leading to an abnormal proliferation of stem-like cells within the germarium.

Potential cystoblast cells in the bam mutant ovaries apparently did not simply remain as stem cells. The small size of the ovary indicated that their ability to proliferate became very limited. Furthermore, cystoblast differentiation was not completely blocked in the mutant because groups of their daughter cells were surrounded by somatic cells to form abnormal cysts in both the ovary and testis. Although we have not looked for the continued presence of ring canals, some of these structures still form between the proliferating cells of other tumorous mutants (King 1970). The residual differentiation observed in the mutant gonads suggested that bam is not required for all the changes associated for cystoblast determination. Genetic tests suggested that the bam mutation we studied was a null allele and did not support the alternative view that the observed 

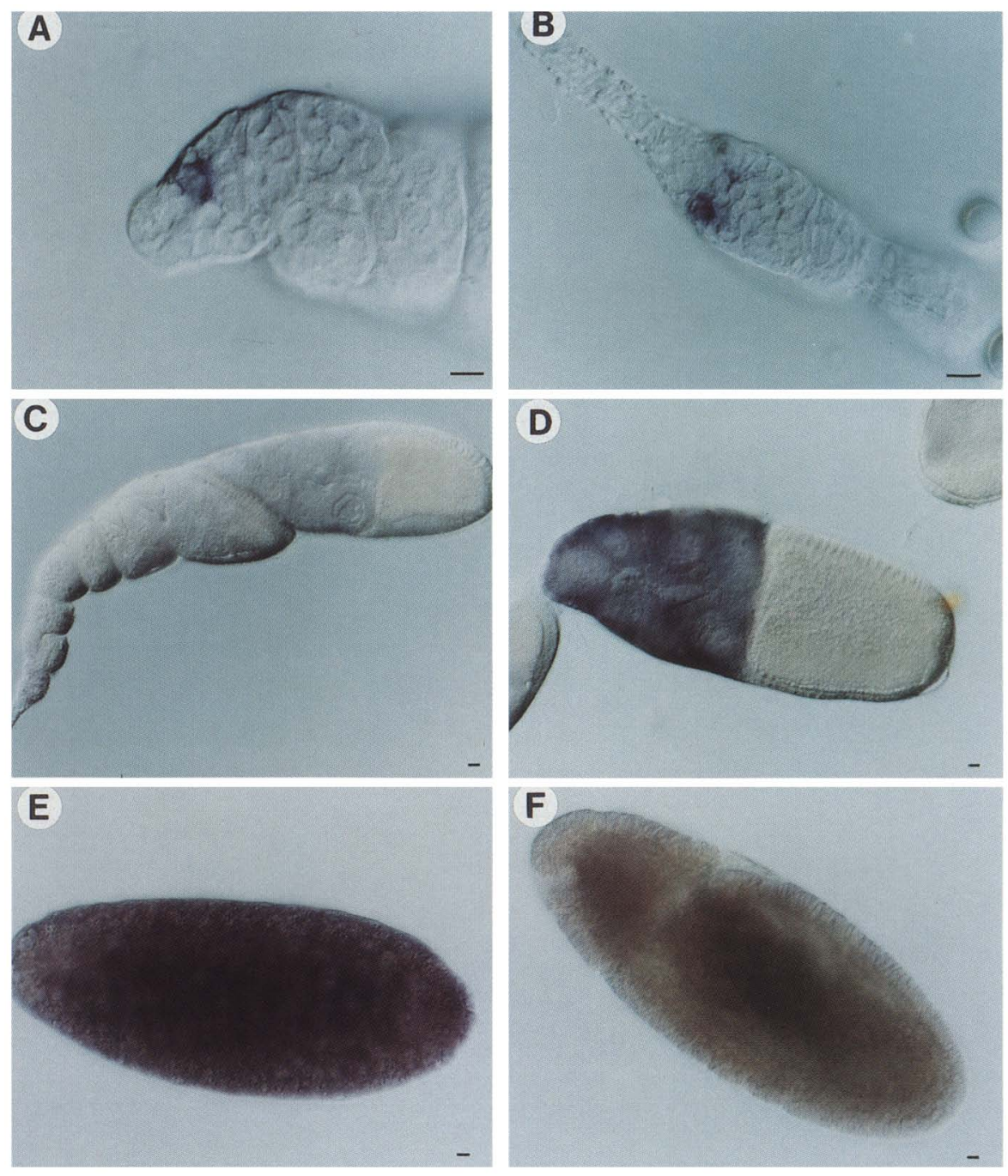

Figure 7. The expression of $b_{a m}{ }^{+}$mRNA in ovaries. Ovaries were dissected from wild-type females and prepared for in situ hybridization by modifications to the methods of Tautz et al. Embryos were collected and prepared as described by Tautz and Pfeifle (1989) (see Materials and methods). Single-stranded antisense RNA probes for bam mRNA were hybridized to ovaries, and stable hybrids were visualized according to methods described by the manufacturer. Egg chamber stages are defined according to King (1970), and embryo stages are defined according to Campos-Ortega and Hartenstein (1985). Positive reactions for bam RNA appear as dark purple in these Nomarski micrographs. $(A$ and $B)$ Germaria: Cells at the location expected for cystoblasts are labeled; $(C)$ ovariole with (unlabeled) egg chambers stages up to stage $10 \mathrm{~A}_{;}(D)$ Stage $10 \mathrm{~B}$ egg chamber: The nurse cells are heavily labeled; $(E)$ embryo, $\sim 1.5 \mathrm{hr}$ : labeling is seen throughout; $(F)$ embryo, $\sim 3.5 \mathrm{hr}$ : No labeling is present above background.

differentiation was caused by a low level of residual bam activity. Molecular studies were also consistent with a null allele; P-element insertion within the bam transcription unit generated an altered mRNA that may no longer be capable of encoding bam function. Recently, we isolated a small deficiency allele of the bam gene that removes the promoter and the amino-terminal third of the putative protein (A. Hilliard and D. McKearin, unpubl.). The phenotype of this deletion allele is essentially indistinguishable from that described for the insertion allele.

A function for bam in establishing the number and character of cystocyte divisions suggests a role for the later expression observed in stage 10 nurse cells. bam
RNA was transported to the oocyte and persisted throughout the first $2 \mathrm{hr}$ of embryonic development. The 13 cleavage divisions that occur during this time resemble cystocyte divisions: The number of divisions is precisely counted, divisions take place within a syncytium, and mitosis is induced prior to a doubling of the cell mass. bam may play a role in the cleavage cell cycle similar to its function during cystoblast divisions. To test this hypothesis, it will be necessary to genetically disrupt bam function after cyst formation, but prior to stage 10 . The ability to rescue bam mutants with transposons containing genomic sequences opens several approaches to this task.

Cystoblast differentiation and cyst formation may not 
occur autonomously. Germ line and somatic cells are known to interact late in oogenesis to pattern the egg chamber and early embryo (Schüpbach 1987; for review, see Manseau and Schüpbach 1989|. However, germ-line cells require early signals from gonadal mesoderm cells to undergo sexual differentiation (see Nöthiger et al. 1989). Two observations suggested that bam acts only in germ-line cells. bam RNA was detected only in the gonad by Northern analysis and only in germ-line cells of the gonad by in situ hybridization. Furthermore, "enhancer-trap" transposons that normally express $\beta$-galactosidase only in ovarian germ-line cells were activated in the cells inside bam egg chambers (not shown). Construction of mosaic ovaries will be necessary to confirm the suggestion of these experiments that bam function is germ line autonomous.

\section{Ovarian tumor genes and germ-line sex determination}

The pathway of sexual differentiation in Drosophila differs between somatic and germ-line cells (for review, see Pauli and Mahowald 1990|. Sex determination in germ-line cells is partially nonautonomous; signals from the somatic gonodal mesoderm are thought to be required to initiate and/or maintain the sexually differentiated state. The master regulatory gene $S x l$ is needed for this signaling (Schüpbach 1985; Steinmann-Zwicky et al. 1989|. In the absence of $S x l, X / X$ stem cells produce tumorous cysts in the ovary that contain cells transformed toward primary spermatocytes. These observations suggested that correct sexual identity is required for cyst development and that the tumorous ovary phenotype may indicate a failure of germ-line sex determination. At least two other tumorous ovary genes, snf and $o v O^{D}$, genetically interact with $S_{X} l$ and are proposed to carry out additional steps in germ-line cell sex determination (Oliver et al. 1987; Steinmann-Zwicky 1988; Oliver et al. 1990).

The similarity between the tumorous ovaries that result from bam mutation to those caused by $S x l$, snf, or ovo mutants suggested an alternative model for bam function, in which the gene carries out a role in germline sex determination. Because bam is expressed and is functionally required, only in the gonads, it would have to encode a function limited to germ-line sex determination, unlike $S_{x l}$ or snf. Because bam is required for male as well as female differentiation, it might act downstream of the previously characterized genes to execute male-specific or female-specific functions, a role analogous to that of doublesex in somatic cells. However, the limitation of bam expression to cystocytes and stage $10 \mathrm{~B}$ egg chambers and the failure to detect different male and female transcripts favor the view that bam controls cell divisions directly, rather than indirectly through effects on sexual differentiation.

If bam does function to control cystoblast divisions, why is the phenotype in the ovary so similar to tümorous ovary genes that disrupt sex determination? We propose that this phenotype is a general sign that cellular interactions organizing the germarium have been disrupted. These interactions may be required to maintain as well as to establish the normal differentiation of cells within the gonads, including their sexual differentiation. Any mutation disrupting the relative number and position of cells might therefore have extensive pleiotropic consequences. When cystoblast differentiation fails due to bam mutation, germ-line cells in the germarium may be plunged into sexual limbo, producing transformed and intersexual cells. Tumorous ovarioles would therefore represent germaria in which communication between germ line and soma has broken down, or where it occurs sporadically and to a limited degree. Only a fraction of the genes that have been shown to mutate to this phenotype would represent sex determination functions, whereas others would carry out roles necessary to maintain normal cell number, position, and communication within the germarium.

\section{Relationship between bam and otu}

The middle half of the bam protein contained regions with weak but significant similarity to the product of the otu locus. This structural similarity and their nearly identical phenotypes in females suggested that the two genes might be functionally related. Like bam, otu is expressed specifically in developing egg chambers and in the testis (Mulligan et al. 1988). otu RNA accumulates in mature eggs (although with different kinetics from bam-see Parks et al. 1986; Parks and Spradling 1987) and may encode an embryonic function because otu ${ }^{l l}$ homozygous females produce embryos that die at an early stage (see Storto and King 1988). At present, there is no evidence that otu functions in the testis /Steinhauer et al. 1989|, although a redundant function cannot be ruled out. Nonetheless, our results suggest that there may be (at least) two classes of tumorous egg chamber genes-genes required for germ-line sex determination like $S x l$, snf, and ovo, and those like bam and otu that participate in early steps in gametogenesis and embryogenesis.

\section{Materials and methods}

Fly stocks

Flies were maintained on yeasted cornmeal/molasses media in bottles or vials as appropriate. Whenever selection for the NeO marker was required, as in recombination experiments for stock construction, G418 was added to the media to a final concentration of $600 \mu \mathrm{g} / \mathrm{ml}$, as described in Cooley et al. (1988). The bam mutation was recovered from the original single P-element mutagenesis carried out by Cooley et al. (1988), and the specifics of the chromosomes and methods used to induce the mutation are described in that reference. All previously reported genetic locus designations are described in Lindsley and Zimm (1985, 1990). Transformation was carried out as described (Spradling 1986).

\section{Cytology}

Microscopy to evaluate the morphology of tissues of homozygous bam and wild-type animals was performed on a Zeiss Axiophot microscope equipped with phase and differential in- 
terference contrast objectives at magnification values of $20-100 \times$. In the case of ovaries, tissue was dissected in saline and fixed with $2 \%$ formaldehyde or viewed unfixed, and ovarioles were teased apart to reveal the egg chambers. These preparations were mounted under a coverslip in excess saline or in saline $/ 25 \%$ glycerol to prevent excessive distortion by compression. When the goal was to release cells from tumorous egg chambers to investigate their morphology under high magnification, individual chambers were manually disrupted or buffer was blotted from under the coverslip to force rupture of chambers by the pressure of the coverslip. These same techniques were used to examine testes.

\section{Isolation of and hybridization to nucleic acids}

DNA was isolated from Drosophila adults according to Cooley et al. (1988). Isolation of RNA and DNA from ovaries was as described previously (Parks et al. 1986). Embryos were collected on rich molasses plates and harvested at specified times to provide a stage-specific profile of embryonic transcripts. To isolate embryo RNA, dechorionated embryos were ruptured in a Teflon/glass homogenizer in buffer $\mathrm{RHB}$, incubated at $37^{\circ} \mathrm{C}$ with proteinase $\mathrm{K}(\sim 100 \mu \mathrm{g} / \mathrm{ml})$ for $1 \mathrm{hr}$, and extracted with phenol/chloroform $(50: 50)$ until the interface was clear (buffer RHB: $25 \mathrm{~mm}$ Tris $(\mathrm{pH} 7.5) ; 20 \mathrm{~mm}$ EDTA $(\mathrm{pH} 8.0) ; 1 \%$ SDS; $0.25 \mathrm{M} \mathrm{NaCl} ; 5 \mathrm{mg} / \mathrm{ml}$ heparin|. Nucleic acids were precipitated by the addition of 2 volumes (DNA) or 2.5 volumes (RNA) of ethanol, precipitates were collected by centrifugation, and pellets were washed with $75 \%$ ethanol.

Restriction enzyme and DNA modification enzyme (Boehringer Mannheim, New England Biolabs) incubations were performed according to manufacturer specifications. Poly|A|-containing RNA was purified on oligo|dT)-cellulose resin (Collaborative Research, type 3 ) by the batch methods described by the manufacturer and in Sambrook et al. (1989). Southern and Northern blotting procedures using GeneScreen nylon membrane, and labeling of DNAs by random hexamer priming were as described in Sambrook et al. [1989).

Hybridizations using DNA probes and subsequent washing protocols were performed at $65^{\circ} \mathrm{C}$ using the procedure of Church and Gilbert as described in Sambrook et al. (1989). Hybridizations using RNA probes were carried out at $65^{\circ} \mathrm{C}$ lexcept where noted in one instance) in $50 \%$ formamide, $5 \times \mathrm{SSPE}^{2} 5 \times$ Denhardt's solution, $1 \%$ SDS, $250 \mu \mathrm{g} / \mathrm{ml}$ denatured DNA, and $250 \mu \mathrm{g} / \mathrm{ml}$ yeast RNA. Final washes were carried out in $25 \mathrm{~mm}$ sodium phosphate (pH 6.8), 1\% SDS, 2 mM EDTA $(\mathrm{pH} 8.0)$, and $0.1 \%$ nonfat dry milk at $68^{\circ} \mathrm{C}$.

\section{RNase protection assays}

Uniformly labeled RNA probes were synthesized from cloned genomic DNA by bacteriophage RNA polymerases. Assays were carried out essentially as described by Sambrook et al. (1989). Each assay contained $0.5 \mathrm{ng}$ of probe $15 \times 10^{8}$ to $8 \times 10^{8}$ $\mathrm{cpm} / \mu \mathrm{g} \mid$ and $10 \mu \mathrm{g}$ of poly $(\mathrm{A})^{+}$RNA from the sample to be assayed. Positive controls were known masses of antisense bam transcripts derived from a bam cDNA clone; these also provide an internal quantification standard. Protection of probe by yeast RNA was included in all sets of assays as a negative control. Protected fragments remaining after digestion were separated on sequencing gels and analyzed by autoradiography of the dried gels.

\section{DNA sequencing and sequence analysis}

DNA was sequenced by the methods of dideoxynucleotide-de- pendent chain termination either from nested deletions of plasmids or by initiation at oligonucleotides complementary to previously determined sequences. Sequences obtained were analyzed on the Staden Nucleic Acid programs on the VAX computer at Johns Hopkins University. Data base searches of the nucleic acid and predicted protein sequences were carried out on the same computer system.

\section{In situ hybridizations to ovaries and embryos}

Developmentally staged embryos were prepared and fixed as described in Tautz and Pfeifle (1989). Ovaries were dissected in EBR and fixed according to modifications provided by S. Parks and $\mathrm{R}$. Lehmann. Briefly, tissue was fixed in $4 \%$ paraformaldehyde, $10 \%$ DMSO, and $1 \times$ PBS with 3 volumes of heptane for $30 \mathrm{~min}$ at room temperature. Tissue was then transferred to $4 \%$ paraformaldehyde $/ 1 \times$ PBS for additional fixation for $20 \mathrm{~min}$ at room temperature. Subsequent steps were as described in Tautz and Pfeifle (1989) beginning with methanol dehydration.

bam antisense RNA probes were prepared using the digoxigenin RNA labeling kit from Boehringer Mannheim following the manufacturer's instructions (RNA Genius Kit). Before use in the hybridization reactions, the average size of the probe was reduced to $\sim 75$ nucleotides following the protocol of Cox et al. (1984). Hybridization was performed in $50 \%$ formamide, $5 \times$ SSC, $100 \mu \mathrm{g} / \mathrm{ml}$ denatured DNA, $50 \mu \mathrm{g} / \mathrm{ml}$ heparin, $0.1 \%$ Tween, and $160 \mu \mathrm{g} / \mathrm{ml}$ yeast tRNA at $53^{\circ} \mathrm{C}$ for $14-18 \mathrm{hr}$. Posthybridization washes and hybrid detection were as described in Tautz and Pfeifle (1989).

\section{Acknowledgments}

We gratefully acknowledge P. Ripoll for the $D f(3 R) L 16$ deficiency, R. Kelley for the cosmid library, and M. Champe for the ovary cDNA library. Thanks go to $M$. Fuller for pointing out that bam spermatocytes do not grow. Thanks also go to C. Hilliard for excellent technical assistance with in situ hybridizations. D.M.M. is especially grateful to Lynn Cooley for the original identification of the bam mutation and for many fruitful discussions. D.M.M. was a Fellow of the American Cancer Society and the Carnegie Institution. This work was further supported by grants from the National Institute of Health and Howard Hughes Medical Institute to A.C.S.

The publication costs of this article were defrayed in part by payment of page charges. This article must therefore be hereby marked "advertisement" in accordance with 18 USC section 1734 solely to indicate this fact.

\section{References}

Campos-Ortega, J.A. and V. Hartenstein. 1985. The embryonic development of Drosophila melanogaster. Springer-Verlag. New York.

Cavener, D.R. 1987. Comparison of the consensus sequence flanking translational start sites in Drosophila and vertebrates. Nucleic Acids Res. 15: 1353-1361.

Cooley, L., R. Kelley, and A.C. Spradling. 1988. Insertional mutagenesis of the Drosophila genome with single P elements. Science 239: 1121-1128.

Cox, K.H., D.V. DeLeon, L.M. Angerer, and R.C. Angerer. 1984. Detection of mRNAs in sea urchin embryos by in situ hybridization using asymmetric RNA probes. Dev. Biol. 101: 485-502.

DiBenedetto, A.G., D.M. Lakich, W.D. Kruger, J.M. Belote, B.S. Baker, and M.F. Wolfner. 1987. Sequences expressed sex-spe- 
cifically in Drosophila melanogaster adults. Dev. Biol. 119: $242-251$.

Gateff, E. 1982. Gonial cell neoplasm of genetic origin affecting both sexes of Drosophila melanogaster. In Embryonic development, cellular aspects (ed. R. Weber and M. Burger), pp. 621-632. Alan Liss, Inc., New York.

Gonzalez, C., I. Molina, I. Casal, and P. Ripoll. 1989. Gross genetic dissection and interaction of the chromosomal region 95E; $96 \mathrm{~F}$ of Drosophila melanogaster. Genetics 123:371377.

Horvitz, H.R. 1988. Genetics of cell lineage. In The nematode Caenorhabditis elegans. Cold Spring Harbor Laboratory, Cold Spring Harbor, New York.

Kemphues, K.J., T.C. Kaufman, R.A. Raff, and E.C. Raff. 1982. The testis-specific $\beta$-tubulin subunit in Drosophila melanogaster has multiple functions in spermatogenesis. Cell 31: $655-670$.

King, R.C. 1970. Ovarian Development in Drosophila melanogaster. Academic Press, New York.

King, R.C. and P.D. Storto. 1988. The role of the otu gene in Drosophila oogenesis. BioEssays 8: 18-23.

Lindsley, D.L. and K.T. Tokuyasu. 1980. Spermatogenesis. In The genetics and biology of Drosophila /ed. M. Ashburner and T.R.F. Wright), vol. 2d, pp. 225-294. Academic Press, New York.

Lindsley, D.L. and G. Zimm. 1985. The genome of Drosophila melanogaster. Dros. Inf. Serv. 62.

. 1990. The genome of Drosophila melanogaster. Dros. Inf. Serv. 68.

Mahowald, A.P. and M.P. Kambysellis. 1980. Oogenesis. In The genetics and biology of Drosophila (ed. M. Ashburner and T.R.F. Wright), vol. 2d, pp. 141-224. Academic Press, New York.

Manseau, L. and T. Schüpbach. 1989. The egg came first of course Trends Genet. 5: 400-405.

Michiels, F., A. Gasch, B. Kaltschmidt, R. Renkawitz-Pohl. 1989. A 14-bp promoter element directs the testis specificity of the Drosophila $\beta 2$-tubulin gene. EMBO /. 8: 1559-1565.

Mulligan, P.K., J.D. Mohler, and L.J. Kalfayan. 1988. Molecular localization and developmental expression of the otu locus of Drosophila. Mol. Cell. Biol. 8: 1481-1488.

Murray, A.W. and M.W. Kirschner. 1989. Dominoes and clocks: The union of two views of the cell cycle. Science 246: 614621.

Nöthiger, R., M. Jonglez, M. Leuthold, P. Meier-Gerschwiler, and T. Weber. 1989. Sex determination in the germ line of Drosophila depends on genetic signals and inductive somatic factors. Development 107: 505-518.

Oliver, B., N. Perrimon, and A.P. Mahowald. 1987. The ovo locus is required for sex-specific germ line maintenance in Drosophila. Genes Dev. 1:913 923 .

- 1988. Genetic evidence that the sans fille locus is involved in Drosophila sex determination. Genetics 120: $159-171$.

1990. Genetic evidence that the ovo locus is involved in Drosophila sex determination. Genetics 125: 535-550.

Parks, S. and A.C. Spradling. 1987. Spatially regulated expression of chorion genes during Drosophila oogenesis. Genes Dev. 1: 497-509.

Parks, S., B. Wakimoto, and A.C. Spradling. 1986. Replication and expression of an X-linked cluster of Drosophila chorion genes. Dev. Biol. 117: 294-305.

Pauli, D. and A.P. Mahowald. 1990. Germ line sex determination in Drosophila. Trends Genet. 6: 259-264.

Rogers, S., R. Wells, and M. Rechsteiner. 1986. Amino acid sequences common to rapidly degraded proteins: The PEST hypothesis. Science 234: $364-368$.

Sambrook, I., E.F. Fritsch, and T. Maniatis. 1989. Molecular cloning: A laboratory manual. Cold Spring Harbor Laboratory Press, Cold Spring Harbor, New York.

Schüpbach, T. 1985. Normal female germ cell differentiation requires the female $X$ chromosome to autosome ratio and expression of sex-lethal in Drosophila melanogaster. Genetics 109: 529-548.

1987. Germ line and soma cooperate during oogenesis to establish the dorsoventral pattern of egg shell and embryo in Drosophila melanogaster. Cell 49: 699-707.

Spradling, A.C. 1986. P element-mediated transformation. In Drosophila; A practical approach (ed. D.B. Roberts), pp. 175-199. IRL Press, Oxford.

Steinhauer, W.R., R.C. Walsh, and L.J. Kalfayan. 1989. Sequence and structure of the Drosophila melanogaster ovarian tumor gene and generation of an antibody specific for the ovarian tumor protein. Mol. Cell. Biol. 9: 57265732 .

Steinmann-Zwicky, M. 1988. Sex determination in Drosophila: The X-chromosomal gene liz is required for $S_{X l}$ activity. EMBO /. 7: 3889-3898.

Steinmann-Zwicky, M., H. Schmid, and R. Nöthiger. 1989. Cell-autonomous and inductive signals can determine the sex of the germ line of Drosophila by regulating the gene Sxl. Cell 57: 157-166.

Storto, P.D. and R.C. King. 1988. Multiplicity of functions for the otu gene products during Drosophila oogenesis. Dev. Genet. 9: 91-120.

Tautz, D. and C. Pfeifle. 1989. A non-radioactive in situ hybridization method for localization of specific RNAs in Drosophila embryos reveals translational control of the segmentation gene hunchback. Chromosoma 98: 81-85.

\section{Note added in proof}

Sequence data described in this paper have been submitted to EMBL/GenBank Data Libraries under accession number $\mathrm{X} 56202$. 


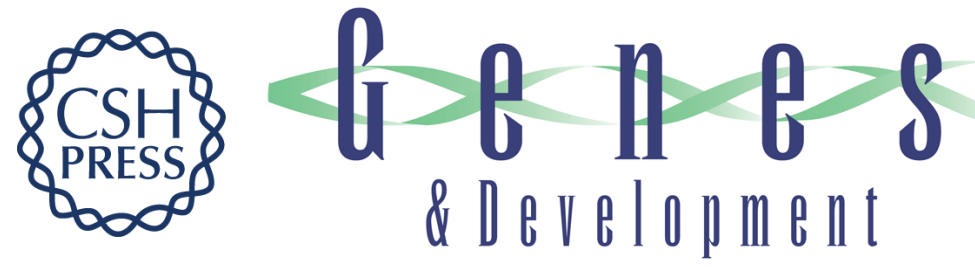

\section{bag-of-marbles: a Drosophila gene required to initiate both male and female gametogenesis.}

D M McKearin and A C Spradling

Genes Dev. 1990, 4:

Access the most recent version at doi:10.1101/gad.4.12b.2242

References This article cites 26 articles, 12 of which can be accessed free at:

http://genesdev.cshlp.org/content/4/12b/2242.full.html\#ref-list-1

License

Email Alerting

Service

Receive free email alerts when new articles cite this article - sign up in the box at the top right corner of the article or click here.

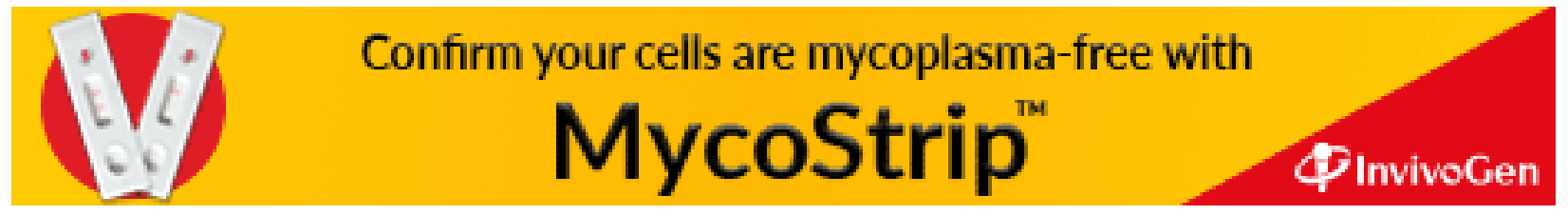

